\title{
Calidad percibida de la atención en salud en una red pública del municipio de Pasto, Colombia
}

\author{
Perceived quality of health care in a public network in the municipality of Pasto, Colombia
}

\author{
María Clara Yépez-Chamorro1,2* orcid.org/0000-0002-3482-5786 \\ Melissa Ricaurte-Cepeda ${ }^{1,2}$ orcid.org/0000-0003-0055-5375 \\ Daniel Marcelo Jurado-Fajardo2,3 orcid.org/0000-0003-0628-0253
}

1 Programa de Promoción de la Salud, Facultad de Ciencias de la Salud. Universidad de Nariño. Pasto, Colombia

2 Centro de Estudios en Salud - CESUN, Universidad de Nariño. Pasto, Colombia

3 Programa de Medicina, Facultad de Ciencias de la Salud. Universidad de Nariño. Pasto, Colombia

Fecha de recepción: Enero 16-2018 Fecha de revisión: Marzo 16-2018 Fecha de aceptación: Abril 13-2018

Yépez-Chamorro MC, Ricaurte-Cepeda M, Jurado DM. Calidad percibida de la atención en salud en una red pública del municipio de Pasto, Colombia. Univ. Salud. 2018;20(2):97-110. DOI: http://dx.doi.org/10.22267/rus.182002.114

\section{Resumen}

Introducción: Las percepciones de los usuarios frente a los servicios de salud son indicadores indispensables de calidad sobre procesos de atención sanitaria. Objetivo: Conocer las percepciones de los usuarios de una red pública del municipio de Pasto, Colombia frente a la calidad de los servicios de salud del primer nivel de atención. Materiales y métodos: Investigación cualitativa con enfoque histórico hermenéutico con 28 participantes, usuarios de centros de atención rurales y urbanos. Las técnicas de recolección de información desarrolladas fueron entrevistas semiestructuradas y un grupo focal. El proceso investigativo abordó las dimensiones: confianza, fiabilidad, responsabilidad, capacidad de respuesta y tangibilidad. Resultados: En las dimensiones se identificaron como limitantes en la calidad, dificultades de acceso por aspectos administrativos y geográficos, problemas en la oportunidad en la atención especializada, fallas en la referencia y contra referencia y la necesidad de generar mejoras en la infraestructura de las IPS. Se reconoció que el buen trato del personal asistencial es un aspecto que incide de manera positiva en la percepción de la calidad. Conclusiones: Mejorar la calidad de los servicios sanitarios, requiere reconocer a los usuarios como el centro de la atención, con miras a la integralidad, continuidad, eficacia, eficiencia y la equidad de los servicios, bajo el imperante fundamental de la garantía y protección del derecho a la salud y a la vida digna de los pacientes.

Palabras clave: Percepción social; calidad de la atención de salud; prestación de atención de salud; pacientes; equidad; servicios de salud. (Fuente: DeCS, Bireme).

\begin{abstract}
Introduction: Perceptions of users from the health services are indispensable indicators for quality on the processes of health care. Objective: To know the perceptions of the users of a public network of the municipality of Pasto, Colombia versus the quality of health services at the first level of attention. Materials and methods: A qualitative research with a hermeneutic historical approach was conducted with 28 participants, who were users of rural and urban centers of care. The techniques developed for collecting the information were semi-structured interviews and a focal group. The research process addressed five dimensions: trust, reliability, responsibility, responsiveness and tangibility. Results: In the dimensions mentioned before, the difficulties of access by administrative and geographical aspects, problems in the opportunity in the specialized attention, faults in the reference and counter-reference and the necessity of infrastructure improvements in the IPS were identified as limitations in the quality. Moreover, it was recognized that the good treatment of the healthcare personnel is an aspect that affects in a positive way in the perception of the quality. Conclusions: Improving the quality of health services, requires recognizing users as the
\end{abstract}


center of attention, with a view to the integrality, continuity, efficiency, efficiency and fairness of services, under the fundamental principle of guarantee and protection of the right to health and life worthy of patients.

Keywords: Social perception; quality of health care; delivery of health care; patients; equity; health services. (Source: DeCS, Bireme).

\section{Introducción}

El estudio de la calidad es un área de interés debido al impacto que genera en las instituciones en términos de reducción de costos, lealtad de los clientes, rentabilidad, entre otros(1). En este contexto, los servicios de salud han acogido los procesos de calidad como un mecanismo que favorece las condiciones de competencia entre proveedores, y con ello, garantizar su rentabilidad y sostenibilidad.

El Sistema General de Seguridad Social en Salud en Colombia (SGSSS) se establece como un modelo integral basado en un esquema de aseguramiento, que permite a instituciones tanto públicas como privadas administrar y prestar el servicio de salud, sus componentes esenciales son: el régimen contributivo (RC) que afilia a los trabajadores asalariados, los trabajadores independientes $\mathrm{y}$ pensionados con ingresos iguales o superiores a un salario mínimo y el régimen subsidiado (RS) que afilia a todas las personas sin posibilidad de pago y que son identificadas por los entes municipales mediante la aplicación de la encuesta del Sistema de Identificación y Clasificación de Potenciales Beneficiarios para los Programas Sociales (SISBEN), así como por los listados censales. La afiliación al SGSSS es obligatoria y se hace a través de las Entidades Promotoras de Salud (EPS), públicas o privadas, que se encargan de afiliar y registrar a los usuarios, recaudar las cotizaciones, organizar y garantizar la prestación del Plan Obligatorio de Salud (POS)(2).

Los proveedores de atención son las Instituciones Prestadoras de Servicios (IPS), que pueden estar o no integradas a las EPS, pero en todo caso son contratadas por éstas; las IPS pueden ser públicas como las Empresas Sociales del Estado (ESES) o privadas como los consultorios, laboratorios, hospitales $\mathrm{y}$ todos los profesionales que, individualmente o agrupados, ofrecen sus servicios de atención a la salud.

Frente a la calidad en la atención, el Estado colombiano ha establecido diferentes disposiciones normativas para la prestación de los servicios de salud, entre ellas: la reforma de la salud a través de la Ley 100 de 1993 que reconoció la calidad como un aspecto fundamental en la atención; el Decreto 1011 de 2006 por el cual se creó el Sistema Obligatorio de Garantía de Calidad de la Atención de Salud del Sistema General de Seguridad Social en Salud, que está constituido por el Sistema Único de Habilitación, la Auditoría para el Mejoramiento de la Calidad de la Atención de Salud, el Sistema Único de Acreditación y el Sistema de Información para la Calidad; la Ley Estatutaria 1751 de 2015 que en su Artículo 6 señala que la calidad e idoneidad profesional son elementos esenciales para asegurar el goce efectivo del derecho a la salud, reconociendo la importancia de centrar los establecimientos, los servicios y las tecnologías en el usuario, a través del fortalecimiento de la formación del personal asistencial, la investigación científica y generación de procesos de evaluación de la calidad, y la Resolución 256 de 2016 que establece disposiciones relacionadas con el Sistema de Información para la Calidad y define los indicadores para monitorear la calidad en salud(3-6).

De igual manera, la Superintendencia Nacional de Salud, entidad del Estado orientada a "la protección del derecho de los usuarios del Sistema General de Seguridad Social en Salud mediante la inspección, vigilancia, control y el ejercicio de la función jurisdiccional y de conciliación", que tiene entre otras funciones, velar por que la atención se realice bajo condiciones de disponibilidad, accesibilidad y calidad(7). 
Aunque el país ha avanzado en materia de políticas para propender por la calidad en la atención, aún persisten dificultades generadas por el "incumplimiento de las obligaciones por parte de las EPS, negación de servicios y exceso de trámites como barrera de acceso para exámenes y servicios médicos especializados", destacándose las limitaciones de acceso para las poblaciones rurales, precariedad de la infraestructura de las instituciones de salud y limitaciones en la disponibilidad de los profesionales ${ }^{(8)}$.

El concepto de calidad del servicio ha sido ampliamente debatido, sus primeras concepciones se orientaron a definirlo desde criterios medibles y verificables centrados en la oferta, enfoque que se conoce como calidad objetiva. Bajo la necesidad de generar cambios a esta concepción, a partir de los trabajos de Grönroos en 1982 y 1994, se enfatizó en la importancia de la interacción activa entre el comprador y el proveedor, este enfoque introdujo la importancia de la percepción del usuario en la calidad de los servicios( $(9,10)$. Desde esta perspectiva, la calidad percibida de los servicios hace referencia a las percepciones y consideraciones subjetivas de los usuarios sobre las características objetivas de los servicios(11). Se han realizado diferentes estudios en torno al constructo calidad del servicio orientados a determinar sus dimensiones constitutivas, Donabedian propone tres componentes de la calidad asistencial: (a) el componente técnico que tiene que ver con el uso de la ciencia y la tecnología en el abordaje de un paciente; (b) el componente interpersonal según el cual, las relaciones que se establecen entre las personas deben estar orientadas por normas y valores sociales, influenciadas por los códigos éticos de los profesionales y por las expectativas de las personas, y (c) los aspectos de confort que son todos los elementos del entorno que proporcionan a los usuarios una atención confortable(12).

Parasuraman, et al., plantearon el modelo SERVQUAL, tomando como base el concepto de calidad de servicio percibida para desarrollar un instrumento cuyo propósito fue cuantificar la calidad del servicio a partir del abordaje de cinco dimensiones: confianza, fiabilidad, responsabilidad, garantía y tangibilidad(13,14). (Figura 1).

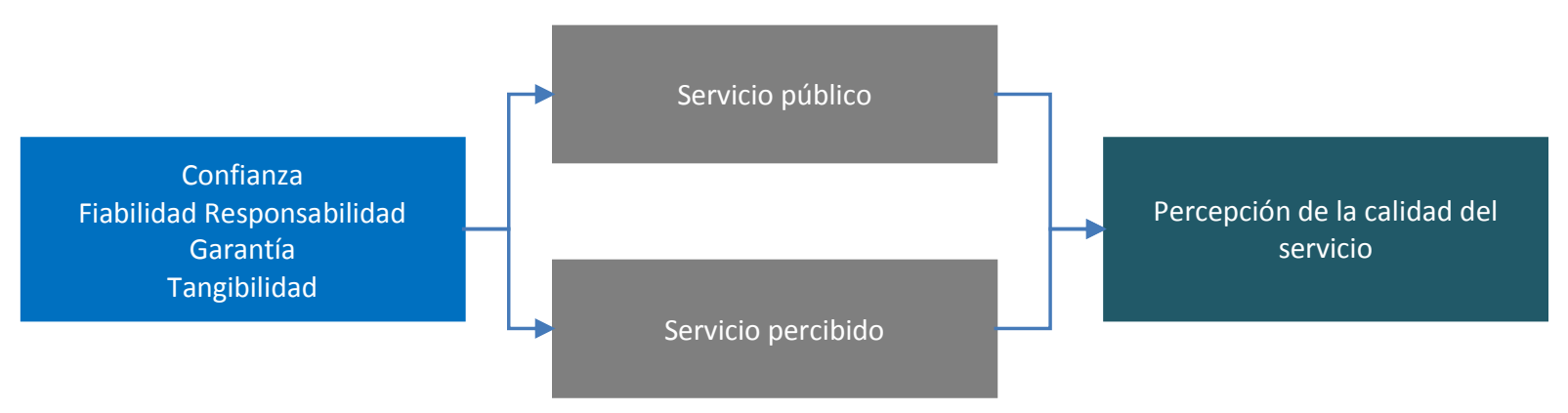

Figura 1. Dimensiones del Modelo SERVQUAL propuesto por Zeithaml, Berry y Parasuraman(14)

Pese a que se han realizado diferentes estudios, no existe consenso frente al número y composición de las dimensiones del constructo calidad del servicio en el área de la salud; sin embargo, gran parte de las propuestas han surgido de las dimensiones planteadas por el modelo SERVQUAL (11).
Teniendo en cuenta las dimensiones propuestas por Parasuraman et al., se desarrolló un estudio cualitativo en el que participaron usuarios del primer nivel de atención del Municipio de Pasto $(13,14)$. Esta investigación tuvo por objeto conocer las percepciones frente a la atención sanitaria recibida con el fin de identificar los aspectos que 
inciden en la calidad desde las experiencias subjetivas de los usuarios, como un aporte a los servicios e instituciones de salud que posibiliten el fortalecimiento de la atención.

\section{Materiales y métodos}

Investigación cualitativa orientada bajo el enfoque histórico hermenéutico, desarrollada a través de un proceso flexible y abierto, centrado en la manera en que los servicios de salud y el proceso de atención son percibidos y experimentados por los usuarios del primer nivel de atención del municipio de Pasto, Colombia(15).

Participaron 28 personas procedentes de zonas rurales y urbanas, mayores de edad (18 años en adelante), pertenecientes a la red pública de

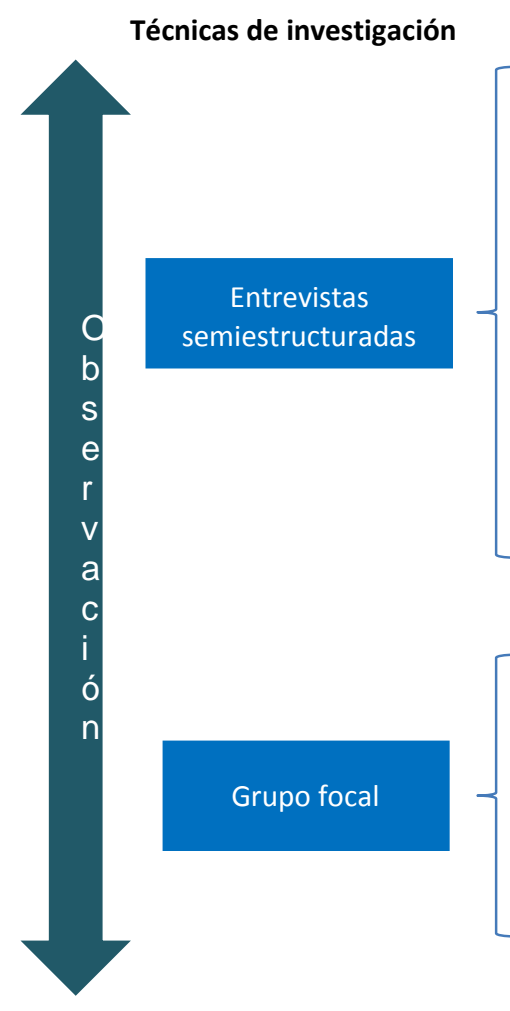

atención, que recibieron servicios médicos en la unidad de urgencias, obstetricia, hospitalización por enfermedad crónica, salud mental y crecimiento y desarrollo, quienes accedieron a participar de la investigación. Los usuarios y su cantidad se seleccionaron por la disponibilidad y el criterio de saturación de información.

Las estrategias de recolección de información desarrolladas fueron: entrevistas semiestructuradas y un grupo focal para explorar las percepciones sobre la calidad en los servicios de atención en salud teniendo en cuenta las cinco dimensiones propuestas por Parasuraman et al., Confianza, Fiabilidad, Responsabilidad, Garantía y Tangibilidad(13). En la Figura 2 se esquematiza el proceso metodológico.

\section{Análisis de la información}

23 participantes

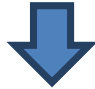

Unidad de urgencias

Consulta por

obstetricia

Hospitalización por

enfermedad crónica

Consulta salud mental

Consulta pediatría

5 participantes

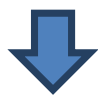

Pacientes con enfermedad crónica

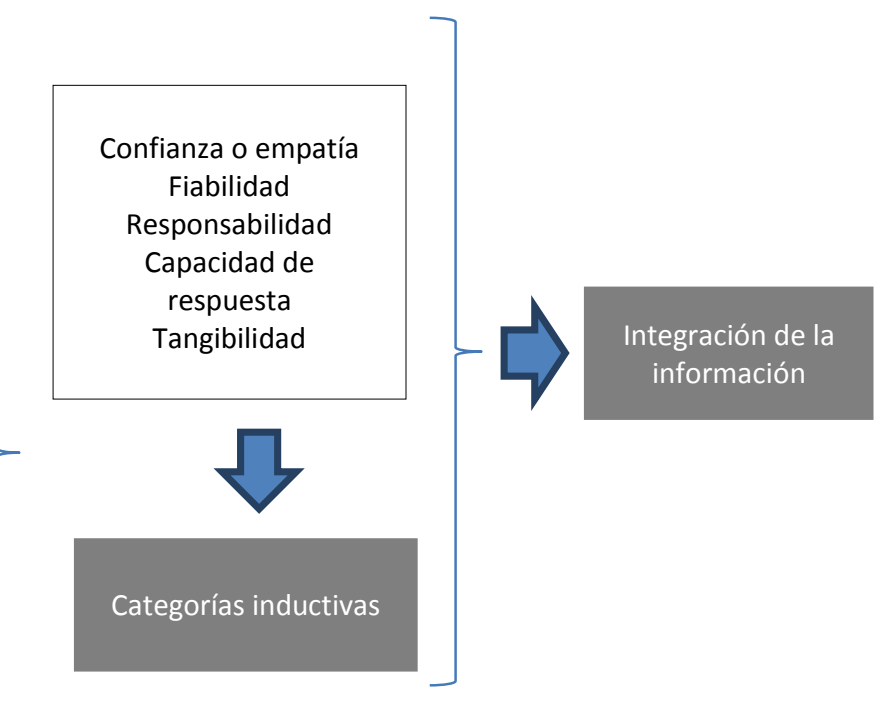

Figura 2. Proceso metodológico del estudio

A partir del análisis de la información, emergieron categorías que posibilitaron ampliar la comprensión de la calidad percibida, se hizo un vaciado constante de información en las categorías inductivas y deductivas. La información fue analizada con el programa Atlas TI. En la Tabla 1 se presentan las categorías que orientaron el estudio. 
Tabla 1. Categorías de análisis estudio

\begin{tabular}{|c|c|c|}
\hline Dimensión & Definición (Parasuraman et al., 1988) & Categorías inductivas \\
\hline Confianza o empatía & $\begin{array}{l}\text { Interés y nivel de atención } \\
\text { individualizada que se puede ofrecer a los } \\
\text { clientes. } \\
\text { (Incluye los criterios de accesibilidad, } \\
\text { comunicación y compresión del usuario) }\end{array}$ & $\begin{array}{l}\text { Oportunidad } \\
\text {-Servicios ambulatorios } \\
\text {-Servicios de urgencias } \\
\text {-Servicios complementarios } \\
\text { Trato } \\
\text {-Identificación de los profesionales } \\
\text {-Claridad de la información } \\
\text {-Información en caso de dudas }\end{array}$ \\
\hline Fiabilidad & $\begin{array}{l}\text { Habilidad para ejecutar un servicio de } \\
\text { forma fiable y cuidadosa }\end{array}$ & $\begin{array}{l}\text {-Tiempo en la atención } \\
\text {-Percepción de la atención sanitaria } \\
\text {-Seguimiento }\end{array}$ \\
\hline Responsabilidad & $\begin{array}{l}\text { Seguridad, conocimiento y atención } \\
\text { brindada a los usuarios y la habilidad del } \\
\text { personal para inspirar credibilidad y } \\
\text { confianza. (Incluye las dimensiones de } \\
\text { profesionalidad, cortesía, credibilidad y } \\
\text { seguridad) }\end{array}$ & $\begin{array}{l}\text {-Características de los profesionales } \\
\text {-Percepción de preparación del personal asistencial } \\
\text {-Confianza }\end{array}$ \\
\hline $\begin{array}{l}\text { Capacidad de } \\
\text { respuesta }\end{array}$ & $\begin{array}{l}\text { Disposición del personal para colaborar a } \\
\text { los usuarios y prestarles un servicio } \\
\text { rápido }\end{array}$ & $\begin{array}{l}\text {-Coordinación entre profesionales } \\
\text {-El proceso de referencia } \\
\text {-La contra referencia } \\
\text {-Coordinación entre profesionales de distinto nivel de } \\
\text { complejidad }\end{array}$ \\
\hline Tangibilidad & $\begin{array}{l}\text { Infraestructura, apariencia de las } \\
\text { instalaciones físicas, equipos, personal y } \\
\text { materiales de comunicación. }\end{array}$ & -Infraestructura, recursos y servicios \\
\hline
\end{tabular}

\section{Consideraciones éticas}

El estudio fue aprobado por el comité de ética de la Universidad de Nariño y se garantizó el respeto a la dignidad, la protección a los derechos humanos y el bienestar de todos los participantes. Hacer parte de esta investigación fue decisión de cada uno de los usuarios, por ello, se cuenta con el consentimiento informado por escrito. De acuerdo a la Resolución 8430 del Ministerio de Salud y Protección social, el estudio se clasificó como una Investigación con riesgo mínimo.

\section{Resultados}

\section{Dimensión confianza}

\section{Oportunidad en la atención: Servicios ambulatorios}

Se identificó que algunos de los participantes consideran que el trámite para la obtención de una cita para los servicios ambulatorios puede ser complicado, debido a que tienen que realizar filas desde tempranas horas de la madrugada para obtener una ficha que posibilite su atención: " $L a$ verdad antes era más difícil porque se debía madrugar a las 4 o 5 de la mañana, ahora han puesto dos horarios, uno a las 7 de la mañana y el otro de 9:30 a 10, pero de todas maneras hay que madrugar mucho para alcanzar". Esta dificultad es atribuida a la cantidad de personas que requieren de atención, así como a problemas en la organización de las instituciones de salud: "Si desde los vigilantes se organizaran, todo fluiría más rápido, pero todo es un desorden".

Una vez que los usuarios han obtenido el turno, la cita es asignada en algunos casos para el siguiente día o entre 2 a 4 días después de solicitarla: " $A$ veces es de un día pero eso si uno está de buenas, porque si no, unos cuatro días o después".

Frente al tiempo que los pacientes deben esperar hasta ser atendidos por el profesional de salud, algunos usuarios refirieron que puede tardar en 
promedio entre 10 y 20 minutos en promedio. Según un paciente: "Hasta que el médico me atiende tengo que esperar unos 10 minutos, depende del turno". Debido a que las citas son agendadas con anticipación, se sugiere a las personas llegar al menos 30 minutos antes de la hora programada.

Respecto a la oportunidad en las consultas por enfermería, algunos usuarios expresaron que el proceso para acceder a la atención es sencillo debido a que generalmente, no hay congestión en el servicio: "Con enfermería me ha ido muy bien, solo voy al consultorio, la atención es buena y no me demoro nada".

\section{Oportunidad en la atención: Servicios de urgencias}

Algunos usuarios manifestaron que el acceso a la atención por urgencias generalmente es ágil: "Aquí en urgencias me atendieron en más o menos en 20 minutos". No obstante, también se reconoció que el tiempo de espera hasta que se realice la atención depende de la cantidad de personas que requieren del servicio y de la gravedad de la urgencia: " $A$ veces hay que esperar hasta 3 horas para que lo atiendan porque hay mucha gente, uno tiene que llegar medio muerto para que lo atiendan rápido...".

\section{Oportunidad en la atención: Servicios complementarios}

De acuerdo con la información obtenida en el estudio, el tiempo de espera para la realización de pruebas complementarias varía de acuerdo al tipo de examen requerido, por ejemplo, "Si es un examen de sangre sencillo, se realiza al otro día o el mismo día", "una citología se demora entre siete y doce días". Pruebas de mayor complejidad, deben ser autorizadas por las EPS: "hay que ir a dejar los papeles para que se los autoricen $y$ después de 10 a 15 días hay que volver a madrugar para recogerlos, o sea que ahora son dos días los que se pierden".

La entrega de los resultados de las pruebas diagnósticas también depende del tipo de examen, en el caso de las citologías y las ecografías, los resultados pueden ser entregados después de un mes de su realización: "Para las citologías siempre es demorado, los de laboratorio a veces se demoran un mes", "los resultados de una ecografía se demoraron entre 20 días a un mes".

\section{Trato: Identificación de los profesionales}

La mayoría de los usuarios del estudio manifestaron que los profesionales que los atienden en consulta no se presentan, limitando su atención al diagnóstico y prescripción del tratamiento: "No únicamente dicen que tiene y ya siga la consulta y nada más". Expresaron que conocen el nombre del profesional a través de su escarapela, la ficha que se encuentra en las puertas de los consultorios o la firma en la orden médica: "Uno a veces le ve una escarapelita $o$ a veces en la bata tienen el nombre, pero no se presentan".

Las personas que refirieron que los médicos se han identificado en consulta, reconocieron este aspecto como significativo debido a que se sintieron valorados $y$ percibieron en los profesionales una actitud empática que posibilitó confianza para expresarse en la consulta: “El doctor que me atendió dijo mi nombre es tal persona, yo soy la que lo voy a atender, estoy a sus órdenes, eso me hizo sentir bien, tranquila, en confianza para contarle lo que me pasa".

\section{Trato: Claridad de la información}

Para algunos usuarios, la información proporcionada por los profesionales no ha sido expresada en términos de fácil comprensión: "Trato de entenderles, ellos hablan con sus términos profesionales (...) cuando no le entiendo le digo: ¿doctor, usted me puede explicar qué significa lo que me está diciendo? hay médicos que le dicen sí, sino uno se queda con la duda". Esta situación es valorada por las personas como una limitante a su derecho de estar informadas sobre su estado de salud y las posibilidades de recuperación: "Uno necesita saber qué es lo que le pasa, que le hablen claro, pero a veces se queda con la incertidumbre, toca tomarse lo que le manda el médico y ya".

Es posible considerar que la claridad en la información durante el proceso de atención puede ser un aspecto que facilite en los pacientes asumir un rol activo durante la consulta, así como 
también frente al cuidado de su salud y bienestar, ejemplo de ello es lo expresado por una participante: "En un principio a mí no me explicaron, no sabía para qué cuidarme, no lo creía tan necesario, por eso no tomaba los medicamentos hasta que la doctora encontró la tensión alta y me explicó, a partir de ese día soy muy puntual".

\section{Trato: Información en caso de dudas}

La mayoría de los participantes expresaron no tener claro a quién acudir en caso de dudas, algunos refirieron buscar al personal de enfermería: "Cuando tengo dudas voy donde las enfermeras, ellas saben mucho", también manifestaron recurrir a los médicos; no obstante, se reconoció la dificultad de acceso a los profesionales en espacios diferentes a la consulta: "Es posible obtener información durante la atención, pero fuera de ella ya es complicado". De igual manera, "Si surge la duda de qué hacer (...) el médico lo atiende con cita, ninguno atiende sin cita".

La oficina de atención al usuario también se identificó como un espacio al que acuden algunos participantes para resolver sus dudas e inquietudes: "Yo siempre vengo a atención al usuario, le ayudan despejar sus dudas".

Es necesario señalar que algunos participantes expresaron que debido a experiencias en las que percibieron que sus dudas no fueron resueltas de manera adecuada, evitan consultar acerca de sus inquietudes. "A veces le contestan con dos piedras en la mano, por eso mejor ni pregunto".

\section{Dimensión Fiabilidad}

\section{Tiempo de la atención}

Para los participantes el tiempo de la atención médica es en promedio de 15 minutos, no obstante consideran que depende de aspectos como el profesional, el número de usuarios que esperan la atención y los motivos de consulta.

Las personas que manifestaron que el tiempo empleado en la consulta médica no ha sido suficiente, reconocieron que este aspecto generó inseguridad con el diagnóstico y el tratamiento, orientándolos a buscar segundas opiniones.
Según un participante: "Hay unos médicos que son muy acelerados, llegan y ya, rapidito, dicen está bien y ya, por eso he cambiado de médicos". Por su parte, los usuarios que consideran que el tiempo en la atención ha sido el necesario, reconocieron que se han sentido escuchados y que han comprendido con mayor claridad las explicaciones de los profesionales sobre su enfermedad y el tratamiento: "la doctora me atiende con su tiempo, con su paciencia, me entrega su tiempo, me escucha, me oye todo lo que yo le comento y me explica claro todo lo que debo hacer", por tanto, es posible que se incremente la probabilidad de adherencia al tratamiento, y con ello, la recuperación de los pacientes.

\section{Percepción de la atención sanitaria}

La mayoría de los participantes del estudio expresaron que se sienten satisfechos con la atención recibida, esta percepción estuvo asociada principalmente con:

(a) El trato del personal asistencial que genera confianza en los profesionales para el manejo y superación de su enfermedad: "La amabilidad y la forma en que he sido tratada me generan la sensación de que estoy en buenas manos". Asimismo, se reconoció la importancia de ser escuchados en la consulta como un factor que contribuye con su satisfacción: "Me han atendido bien, si me han escuchado y yo también pregunto y ellos me dan las respuestas en el mismo momento".

(b) Los medicamentos recetados y entregados también se identificaron como elementos que inciden en la satisfacción de los usuarios, "Hoy me pareció muy buena la atención porque de una me mandaron hartos medicamentos".

(c) La realización de pruebas complementarias se reconoció como un factor que aporta a la satisfacción de la atención recibida, para algunos usuarios, este aspecto evidencia que las instituciones de salud y sus profesionales disponen todos sus recursos y tecnologías en pro de la salud y el bienestar: "Me dieron lo que necesitaba (...) radiografías, sangre, los exámenes que se tienen que hacer, entonces uno se siente bien". 
(d) La efectividad de los tratamientos: " $\mathrm{La}$ atención es muy buena porque ya no me siento tan enferma, me ha hecho bien todo lo que me mandó el médico". Percibir cambios positivos en la salud fue para los usuarios el principal aspecto que refleja la calidad en la atención recibida.

\section{Seguimiento}

La mayoría de los participantes del estudio refirieron que cada vez que asisten a los centros de salud son atendidos por diferentes profesionales, situación que imposibilita realizar un seguimiento a su tratamiento y proceso de evolución. Aunque los pacientes pueden elegir al profesional para la consulta, la alta rotación del personal asistencial se identificó como un aspecto que dificulta la atención por el mismo médico cada vez que es requerida. "Es que ahora cambian de doctores con una rapidez inimaginable (...) ha habido muy buenos médicos pero cuando uno va la próxima vez, ya no están". Algunos usuarios expresaron que el cambio de profesional en la atención ha implicado suspender los tratamientos iniciados y acoger nuevas orientaciones: "El cambio descontrola, cada médico que a uno lo mira le voltea el manejo y el tratamiento".

De acuerdo con algunos participantes, ser atendido por el mismo médico facilita la construcción de una relación de confianza, la continuidad y seguimiento a los tratamientos e incrementa la posibilidad de una pronta recuperación: "Cuando a uno ya lo conoce su médico, él ya sabe que es lo que uno tiene y lo cura rápido (...) El médico nuevo es nuevo, da miedo hasta preguntarle, uno no sabe cómo es él".

\section{Dimensión responsabilidad}

\section{Percepción de preparación del personal asistencial}

La mayoría de los participantes del estudio consideran que existe un buen nivel de preparación de los profesionales que los atienden, aspecto que asocian con la claridad de la información proporcionada y por el trato que reciben. Frente al nivel de preparación, un participante expresó: "Ahora está bien avanzado, el personal esta eficiente, lo atiende muy bien, están atentos a las preguntas que uno les hace y están inmediato las respuestas". La percepción de falta de preparación y de experiencia genera inseguridad y desconfianza por la atención recibida: "Algunos médicos apenas están iniciando a ejercer y haciendo sus prácticas, no me gusta que me atiendan así".

\section{Características de los profesionales}

Entre las características atribuidas por los usuarios al personal sanitario se encontró: "Son amables", "fueron pacientes", "muy eficientes", "son atentos" y "buenas personas". De igual manera, algunos participantes manifestaron que la carga de trabajo puede incidir en el comportamiento de los profesionales: "A veces son groseros, están aburridos, estresados, depende del trabajo".

\section{Confianza}

A partir de la información obtenida es posible identificar algunos aspectos de la atención que generan confianza en los pacientes, entre ellos: (a) La percepción de preparación del personal asistencial: "Me da tranquilidad que son profesionales, de que es un centro médico que tiene personal bien preparado, bien capacitado"; (b) El trato que reciben: "Cuando una persona que llega uno y lo atiende mal, uno ya empieza como a dudar"; (c) La claridad en las explicaciones sobre la enfermedad y el tratamiento: "Ella me explicó todas las palabras como son y como es, entonces uno se siente bien, uno se siente con confianza con la doctora" y (d) La familiaridad de los profesionales: "Soy desconfiada en urgencias, yo digo este médico será bien o no, en cambio con el de consulta externa uno se siente confiada, ya lo conoce".

Los participantes del estudio expresaron que han percibido respeto por su intimidad reflejado en la manera en que fueron tratados por el personal sanitario: "He sentido respeto por la forma que lo examinan, por la delicadeza, un doctor decía disculpa te voy a hacer esto". Un aspecto que ha generado incomodidad ha sido la presencia de estudiantes practicantes en el momento de la atención "A veces cuando hay practicantes entonces uno se siente incómodo". 


\section{Dimensión capacidad de respuesta}

\section{Coordinación entre profesionales}

Algunos participantes coincidieron en afirmar que perciben coordinación y trabajo en equipo en las IPS a las que acuden, reflejándose en el manejo de la información, la relación de colaboración entre el personal médico y el de enfermería, y el uso de protocolos de atención. "Yo veo que trabajan en equipo, entre ellos se colaboran el uno al otro". De igual manera, se considera que la coordinación entre el personal asistencial es indispensable para garantizar la calidad en la atención: "Tienen que tener coordinación, seguir con un esquema para poder tratar a los pacientes, no pueden llegar con una parte y saltarse a otra sino que tiene que haber un orden".

Aunque se percibe coordinación, algunos pacientes reconocen dificultades que son atribuidas al desempeño individual que puede limitar el trabajo en equipo: "Para que sea coordinado todos deben hacer bien las cosas, pero unos las hacen y otros no, entonces hasta ahí llega el trabajo en equipo (...) a unos trabajadores no les interesa, entonces no hay equipo".

\section{El proceso de referencia}

Todos los participantes que fueron remitidos a atención especializada manifestaron que el proceso para obtener una cita requiere de distintos trámites, entre ellos, la autorización de las EPS. "Cuando lo remiten al especialista toca ir a hacer autorizar (...) uno tiene que madrugar a las 4 ó 5 am (...) uno es parada la mañana entera, se cansa y no rinde, son 500 fichas no dan más". En muchos casos para lograr esta aprobación, los pacientes han realizado filas desde tempranas horas de la madrugada para la entrega de documentos que en ocasiones no fueron proporcionados por las IPS al momento de referirlos, razón por la cual tuvieron que regresar a los centros de atención primaria e iniciar el trámite: "El otro día que vine no me dieron la remisión, me toco volver y hacer nuevamente las filas, como uno no sabe".

Esta situación afecta especialmente a las personas procedentes de las zonas rurales del Municipio, quienes se deben desplazar desde sus lugares de residencia a los sitios dispuestos por las EPS, ubicados en la ciudad. "Yo vivo en un corregimiento, me ha tocado muy duro, toca venirse en la noche para ver si se cuenta con suerte y lo mandan al médico".

Algunos participantes manifestaron que la inseguridad y la desorganización durante las filas para obtener las autorizaciones, son situaciones que ponen en peligro su vida e integridad, según un usuario: "A esa hora es muy peligroso, hasta problemas de puño habido, peleas entre los mismos usuarios, con los vigilantes, con el personal que atienden, es mucho caos".

El acceso a la atención por especialistas fue una de las mayores dificultades manifestadas por los participantes debido a que el trámite no sólo ha implicado la realización de filas para hacer la solicitud con las EPS, sino además, gestionar la autorización y pronta asignación de una cita. Para un participante: "Aquí quienes mandan son las EPS, hay que esperar a que le autoricen y que le den la cita con el médico, eso puede demorar varias semanas o meses".

\section{La contra referencia}

Los participantes que fueron atendidos por especialistas expresaron que no fueron contra referidos a sus centros de atención primaria para realizar el seguimiento: "El doctor me revisó y me mandó el tratamiento y ya, nunca me dijo que vuelva a sacar cita en el centro de salud, no sabía que tenía que hacer eso".

A partir de la información obtenida, es posible considerar que en el circuito de referencia y contra referencia, el proceso de referencia se cumple aunque presenta dificultades generadas por la intermediación de las EPS, caso diferente ocurre con la contra referencia debido a que en muchos casos no se genera, imposibilitando la continuidad en el proceso de atención de los pacientes.

\section{Coordinación entre profesionales de distinto nivel de complejidad}

Algunos usuarios expresaron en las entrevistas que han percibido que no existe coordinación entre los profesionales de atención primaria y atención especializada debido a contradicciones 
en el diagnóstico: "De acá (centro de salud) la mandan por una cosa y allá (hospital de segundo/tercer nivel de complejidad) dicen que no, no hay coordinación". De igual manera, consideran que los sistemas de información no son compatibles entre los diferentes niveles de complejidad, razón por la cual, los usuarios deben proporcionar a los especialistas información ya suministrada en el primer nivel de atención. Al referirse sobre la coordinación entre los profesionales del centro de salud y del hospital de segundo/tercer nivel en los que ha recibido atención, un participante expresó: "Le vuelven a hacer preguntas, a indagar, entonces uno tiene que volver a responder, deberían tener una carpeta o hacer un seguimiento, no hay coordinación".

\section{Dimensión tangibilidad}

\section{Infraestructura, recursos y servicios}

Los usuarios reconocen que la infraestructura del hospital de primer nivel al que acuden ha mejorado, proporcionando mayor comodidad en la atención; no obstante, manifestaron que zonas como la sala de espera en la que se concentran las personas de zonas urbanas y rurales que acuden por los servicios, no es un espacio suficiente, generando aglomeraciones en los pasillos, situación que afecta la movilidad de los pacientes y del personal sanitario: "La salita de espera es muy pequeña para cantidad de gente que entra diariamente aquí, hay que hacerse en cualquier lado". Se percibe que el personal con el que cuenta la institución no es suficiente para la cantidad de personas que requieren de la atención, este aspecto se identificó como una de las principales razones por las cuales los usuarios deben hacer largas filas para lograr un servicio.

De igual manera, algunos usuarios reconocieron como un avance, la digitalización de las historias clínicas, "Ahora tienen su buena tecnología porque antes todo se hacía manual", situación que también ha sido percibida como una limitante debido a las fallas en el sistema de información que ha implicado en algunos casos demoras en el proceso de atención: "Desde ayer que no hay sistema y apenas hoy me dan la información".
Pese a que se trata de un hospital de primer nivel, los usuarios expresaron la necesidad de incorporar nuevas tecnologías y servicios, para que en los casos que lo ameriten, se posibilite la atención evitando las referencias a otros niveles de complejidad. "Hay algunas cosas que faltan, por ejemplo en odontología solamente hacen las calcitas, tuve que hacerme un tratamiento de conducto y para eso me remitieron a otra parte, hasta que me autorizaron, qué problema". Para los participantes, generar mayor resolutividad en el primer nivel de complejidad se evidenció como una alternativa para descongestionar la atención especializada.

\section{Discusión}

La calidad de los servicios de salud ha sido un tema ampliamente abordado, aunque no es posible determinar los criterios o dimensiones para su evaluación, existe consenso frente a la presencia de características objetivas de los servicios de salud indispensables en los procesos de atención. Contrario a esto, los aspectos subjetivos de la experiencia de los usuarios se reconocen con menor frecuencia como un indicador de la calidad. Aunque en el país se ha reconocido a la salud como un derecho humano fundamental, se ha explicitado el propósito de garantizar el acceso y se ha avanzado en la cobertura a nivel nacional, aún se evidencian barreras que afectan la atención y la satisfacción de los usuarios frente a los servicios recibidos(16).

En la presente investigación se identificó que la oportunidad en algunos servicios sanitarios incide en la percepción de calidad debido a que existen barreras en el proceso para la obtención citas, entre ellas, los trámites administrativos ante las EPS, que pueden limitar atención oportuna. De acuerdo con Sánchez, et al., la organización del sistema de salud en Colombia "obliga a los pacientes a recorrer un proceso de trabas y requerimientos administrativos, que incluso involucra el uso de recursos legales"(17).

Los participantes reconocieron que deben enfrentar diferentes barreras para acceder a los servicios de salud generadas por fallas en la comunicación entre EPS e IPS, dificultades en la 
continuidad de los servicios, por demora en las autorizaciones, problemas en la oportunidad para citas con especialistas, procedimientos quirúrgicos, entrega de medicamentos, entre otros, que han ocasionado percepciones negativas, así como efectos perjudiciales en su salud(18). Entre las barreras de acceso identificadas en este estudio, se destacan las limitaciones geográficas especialmente para las personas que habitan en zonas rurales del municipio, quienes deben desplazarse a la ciudad para la obtención de citas y/o realización de exámenes complementarios, generándose gastos adicionales a la atención que deben ser asumidos por los pacientes. Como también la gestión de autorizaciones para acceder a servicios especializados y medicamentos que no están incluidos en el POS.

Las dificultades anteriormente mencionadas que reconocieron los participantes, coinciden con lo identificado en el estudio realizado por GarcíaSubirats, et., al, en el que se analizan los diferentes obstáculos de acceso a los servicios de salud y se establece que las barreras de tipo geográfico, económico y las relacionadas con las autorizaciones por parte de las EPS, son las principales limitantes de la atención(19).

Al igual que los resultados obtenidos en la Encuesta de Evaluación de los Servicios de las EPS 2016, la mayoría de los participantes del estudio refirieron sentirse inconformes con los tiempos de espera para la atención, especialmente en la atención especializada, aspecto que no resulta novedoso frente a los hallazgos de estudios anteriores, como el publicado en el 2009 por Vargas y Molina realizado en seis ciudades de Colombia(20-22). En esta investigación se identificaron como limitaciones en la atención integral: los trámites burocráticos para el acceso a la atención, la insuficiente oferta de especialistas y las barreras para acceder al segundo y tercer nivel, situaciones atribuibles a las aseguradoras y a los prestadores de servicios de salud.

De manera similar, en el Informe Nacional de Calidad de la Atención en Salud 2015 del Ministerio de Salud y Protección Social, se reportó que pese a las dificultades de acceso $y$ oportunidad, los pacientes reconocen que en general, han recibido un trato amable $y$ respetuoso por parte del personal sanitario y administrativo de las instituciones de salud, aspecto que además asocian a la calidad de información que manifestaron recibir(23).

La posibilidad de establecer espacios de comunicación con los profesionales asistenciales se identificó como un elemento que contribuye con la percepción de calidad y genera confianza en la atención recibida. Es necesario considerar que la comunicación posibilita que los pacientes se sientan escuchados, comprendan el significado de su enfermedad y se sientan copartícipes de su atención(24). Britten, et al., encontraron que el intercambio de información entre el médico y paciente, la claridad en el diagnóstico y la comprensión del tratamiento contribuyen con la adherencia terapéutica por parte del usuario(25).

Teniendo en cuenta lo anterior, la relación que establecen los profesionales asistenciales y los pacientes, incide no sólo en la percepción de la calidad de los servicios, sino además, en su estado de salud/enfermedad. Estudios evidencian que la relación y comunicación efectiva entre el médico y el paciente, genera beneficios para la salud física y mental de las personas que requieren de atención, e incrementa la confianza y compromiso de los profesionales ${ }^{(26)}$.

La calidad de la información se relacionó directamente con su claridad, los usuarios que percibieron que las indicaciones de los profesionales sanitarios fueron de fácil comprensión, refirieron sentirse más seguros frente al tratamiento recibido y sus posibilidades de recuperación. En el estudio realizado por Barca et al., se identificó que el hecho de que un profesional sanitario utilice un lenguaje ajeno a sus pacientes, limita la posibilidad de establecer vínculos de confianza, coadyuvando a mantener en los pacientes y sus familiares, temores o dudas sobre su padecimiento y las posibilidades de recuperación(27).

Las percepciones de los usuarios frente al proceso de referencia permitieron comprender la ruta que deben seguir los pacientes para lograr la atención 
desde el primero hasta niveles de mayor complejidad. En este proceso, el papel de las EPS resulta crucial debido a que de ellas depende la aprobación de la atención, la institución que brindará el servicio y el tiempo que deberá esperar un paciente. En el Informe Nacional de Calidad de la Atención en Salud 2015, los usuarios identificaron entre las principales razones por las cuales las EPS negaron un servicio: la no disponibilidad del recurso, el servicio no incluido en el POS y la no contratación con el prestador(23).

Según el Ministerio de Salud y Protección Social, las instituciones de salud tienen que monitorear la oportunidad de la referencia como un indicador que debe conducir a la generación de acciones de mejoramiento que incrementen la posibilidad de que los usuarios reciban los servicios que necesitan sin que se generen retrasos que pongan en riesgo su vida o su salud, aspecto que requiere del seguimiento al cumplimiento de las políticas nacionales en salud, así como del compromiso de las aseguradoras en brindar garantías que permitan la atención oportuna e integral de los ciudadanos(28).

En relación a la contra referencia fue posible reconocer que se trata de un proceso que presenta dificultades debido principalmente a que los especialistas no contra refieren a los pacientes al primer nivel después de su consulta, imposibilitando la continuidad y el seguimiento al proceso de atención. Para que el proceso de atención se pueda generar de manera continua, los niveles de atención deben operar con una adecuada referencia y contrarreferencia, para ello, es fundamental la coordinación entre los diferentes niveles ${ }^{(29) .}$

Aunque no es posible desconocer las condiciones objetivas de la calidad en la prestación de los servicios, las expectativas y experiencias de los pacientes durante la atención, deben ser reconocidas y tomadas en consideración en las decisiones frente a los servicios de salud y la atención, sólo ello garantizará la calidad como proceso que propenda por la garantía de los derechos de los ciudadanos.
Los cambios en la legislación en salud producidos a partir de la Ley Estatutaria 1751 de 2015 y la Política de Atención Integral en Salud (PAIS) por la cual se adopta el Modelo Integral de Atención en Salud (MIAS), generan un nuevo escenario para las instituciones y la atención sanitaria, ampliando el contexto de la salud al introducir las acciones intersectoriales como mecanismo para afectar los determinantes sociales, así como el reconocimiento de la evaluación técnica y social del Sistema de Salud en su efectividad para mejorar las condiciones de salud de las personas $(5,30)$. Se espera que estos avances en Colombia permitan generar cambios en la prestación de los servicios que favorezcan el acceso, la oportunidad, la continuidad y la integralidad en la atención bajo una perspectiva de calidad centrada en la garantía del derecho a la salud y la vida digna de las personas y comunidades.

Teniendo en cuenta que la calidad de la atención sanitaria ha sido ampliamente estudiada bajo enfoques cuantitativos centrados en las características objetivas de los servicios, se resalta la necesidad de reconocer las experiencias de los usuarios y re significar la noción de calidad no como un fin que favorece la competencia en el mercado, sino como un imperante fundamental de todo proceso en el que la vida y el bienestar de un ser humano estén implicados.

\section{Conclusiones}

La calidad es un constructo multidimensional que ha sido abordado desde dos enfoques, uno objetivo, centrado en las variables que inciden en la prestación de los servicios en salud, y otro subjetivo, desde el cual se reconoce la importancia de las expectativas y la experiencia de los pacientes como un indicador indispensable de la calidad.

Si bien no es posible desconocer las condiciones objetivas de la calidad de los servicios, la manera en que el paciente percibe el servicio, debe ser una condición indispensable en todo proceso de calidad, por esta razón se fundamenta la necesidad de realizar estudios que permitan 
aproximarse a las experiencias de los usuarios frente a los procesos de atención sanitaria.

Aunque se identificaron limitantes en la atención como las dificultades de acceso generadas principalmente por aspectos administrativos y geográficos, problemas en la oportunidad especialmente en la atención especializada, fallas en el circuito de referencia y contra referencia, así como la necesidad de generar mejoras en la infraestructura de las IPS, en términos generales, para los participantes del estudio la calidad de los servicios es buena, se reconoció el trato recibido por el personal asistencial como una de las principales razones que contribuyeron con esta percepción.

Los cambios en la forma de concebir la salud y administrar los servicios generados a partir de la Ley Estatutaria 1751 de 2015 y la Política de Atención Integral en salud, proponen un nuevo escenario para la atención, en el que se espera, se superen las dificultades generadas por la intermediación de las EPS, se conciba al usuario como el centro de la atención y se avance hacia la integralidad, la continuidad, la eficacia, la eficiencia y la equidad en la atención, bajo el imperante fundamental de la garantía y protección del derecho a la salud y a la vida digna de los pacientes ${ }^{(30)}$.

\section{Conflicto de interés}

No existen conflictos de interés.

\section{Referencias}

1. Gurau C. Tailoring e-service quality through CRM. Managing Service Quality. 2003;13(6):520-531.

2. Agudelo C, Cardona J, Ortega J, Martínez R. Sistema de salud en Colombia: 20 años de logros y problemas. Ciência \& Saúde Coletiva. 2011;16(6):2817-28.

3. Kerguelén C. Calidad en salud en Colombia. Los principios. Bogotá: Ministerio de la Protección Social; 2008.

4. Ministerio de Salud y Protección Social. Decreto 1011 de 2006. Por el cual se establece el Sistema Obligatorio de Garantía de Calidad de la Atención de Salud del Sistema General de Seguridad Social en Salud. Bogotá: MinSalud; 2006.

5. Congreso de Colombia. Ley Estatutaria de la Salud 1751 de 2015. Por medio de la cual se regula el derecho fundamental a la salud y se dictan otras disposiciones. Bogotá: Congreso de Colombia; 2015
6. Ministerio de Salud y Protección Social. Resolución 256 de 2016. Por la cual se dictan disposiciones en relación con el sistema de información para la calidad y se establecen los indicadores para el monitoreo de la calidad en salud. Bogotá: MinSalud; 2016.

7. Superintendencia Nacional de Salud. Misión corporativa. [Internet]. Bogotá: SuperSalud; 2017. [Citado julio 2017]. Disponible en: https://www.supersalud.gov.co/esco/superintendencia/nuestra-entidad/misi\%C3\%B3ny-visi\%C3\%B3n

8. Ministerio de Salud y Protección Social. Plan Decenal de Salud Pública 2012-2021. Bogotá: MinSalud; 2013.

9. Grönroos C. An applied service marketing theory. European Journal of Marketing. 1982; 16(7):30-41.

10. Grönroos C. Marketing y gestión de servicios: la gestión de los momentos de la verdad y la competencia en los servicios. Madrid: Ediciones Díaz de Santos; 1994

11. Losada M, Rodríguez A. Calidad del servicio de salud: una revisión a la literatura desde la perspectiva del marketing. Cuadernos de Administración. 2007;20(34):237-258.

12. Donabedian A. Evaluating the Quality of Medical Care. The Milbank Q. 2005;83(4):691-729

13. Parasuraman A, Zeithaml V, Berry L. A conceptual model of service quality and its implications for future research. Journal of Marketing. 1985;49(4):41-50.

14. Parasuraman A, Zeithaml V. Berry L. SERVQUAL: A multiple-item scale for measuring consumer perceptions of service quality. Journal of Retailing. 1988;64(1):12-37.

15. Salgado A. Investigación cualitativa: diseños, evaluación del rigor metodológico y retos. LIBERABIT. 2007;13:7178.

16. Restrepo-Zea JH, Silva-Maya C, Andrade-Rivas F, VHDover R. Acceso a servicios de salud: análisis de barreras y estrategias en el caso de Medellín, Colombia [Internet]. Rev. Gerenc. Polít. Salud. 2014;13(27):242-265. [Citado mayo 2017]. Disponible en: http://dx.doi.org/10.11144/Javeriana.rgyps13-27.assa

17. Sanchez G, Laza C, Estupiñan C, Estupiñan L. Barreras de acceso a los servicios de salud: narrativas de mujeres con cáncer de mama en Colombia. Facultad Nacional de Salud Pública. 2014;32(3):305-313.

18. Rodríguez J, Rodríguez D, Corrales J. Barreras de acceso administrativo a los servicios de salud en población Colombiana. Ciência \& Saúde Coletiva. 2015;20(6):1947-1958.

19. Garcia-Subirats I, Vargas I, Mogollón AS, et al. Barriers in access to healthcare in countries with different health systems. A study in municipalities of central Colombia and north-eastern Brazil. Soc Sci Med. 2014;106(1):204213.

20. Ministerio de Salud y Protección Social. Observatorio de Calidad, Encuesta de evaluación de las EPS. Bogotá: Minsalud; 2016. Recuperado de: http://calidadensalud.minsalud.gov.co/Observatorio/ EncuestadeCalidaddeEPS.aspx

21. Ministerio de Salud y Protección Social. Encuesta de Evaluación de los Servicios de las EPS 2013-2014. Bogotá: Minsalud; 2015. 
22. Vargas J, Molina G. Acceso a los servicios de salud en seis ciudades de Colombia: limitaciones y consecuencias. Rev Fac Nac Salud Pública. 2009;27(2):121-130.

23. Ministerio de Salud y Protección Social. Informe Nacional de la Calidad de la Atención en Salud 2015. Bogotá: Minsalud; 2015.

24. Hernández-Torres I, Fernández-Ortega $\mathrm{M}$, IrigoyenCoria A, Hernández-Hernández M. Importancia de la comunicación médico-paciente en medicina familiar. Medicina Familiar. 2006;8(2):137-143.

25. Britten N, Stevenson F, Barry C, Barber N, Bradley C. Misunderstanding in general practice: qualitative study. BMJ. 2000;320(1):484-488.

26. Bascuñán M. Cambios en la relación médico-paciente y nivel de satisfacción de los médicos. Rev. méd. Chile. 2005;133(1):11-16.

27. Herrera-KiengelherI L, Villamil-ÁlvarezI M, PelcastreVillafuerte B, Cano-Valle F. y López-Cervantes M. Relación del personal de salud con los pacientes en la Ciudad de México. Rev. Saúde Pública. 2009;43(4):1-5.

28. Ministerio de Salud y Protección Social. Atributos de la Calidad en la Atención en Salud [internet]. Bogotá: MinSalud; 2018. [Citado julio 2017]. Disponible en: https://www.minsalud.gov.co/salud/Paginas/ATRIBU TOS-DE-LA-CALIDAD-EN-LA-ATENCI\%C3\%93N-ENSALUD.aspx

29. Vignolo J, Vacarezza M, Álvarez C, Sosa A. Niveles de atención, de prevención y atención primaria de la salud. Arch Med Interna. 2011;33(1):11-14.

30. Ministerio de Salud y Protección Social. Política de Atención Integral en Salud "Un sistema de salud al servicio de la gente” Bogotá: Minsalud; 2016. 